\title{
ON THE POINT-LINE AS ELEMENT OF SPACE: A STUDY OF
}

\section{THE CORRESPONDING BILINEAR CONNEX*}

BY

\section{EDWARD KASNER}

In the last paper published during his lifetime, CleBsch $\dagger$ enriched the analytical geometry of the plane by the introduction of a new form, the connex, which includes as very special cases both the curve considered as point locus and the curve considered as line envelope. The connex $(m, n)$, of the $m$-th order and $n$-th class, is represented by an equation $f^{\prime}\left(x_{1}, x_{2}, x_{3} ; u_{1}, u_{2}, u_{3}\right)=0$, involving a set of point coördinates and a set of line coördinates. It may be defined as a manifold of $\infty^{3}$ elements each consisting of a point and a line. Clebsch studied the case $(1,1)$, equivalent to a collineation; and with his pupil GodT, the case $(1, n) . \ddagger$ The general connex $(m, n)$ has been only incom-. pletely investigated, principally in connection with the theory of algebraic differential equations. $\S$

An extension to space was first proposed by KRAUSE, $\|$ who took for element the combination of a point and plane. An equation

$$
f\left(x_{1}, x_{2}, x_{3}, x_{4} ; u_{1}, u_{2}, u_{3}, u_{4}\right)=0
$$

represents a manifold of $\infty^{5}$ elements which may be termed for distinction a point-plane connex. KRAUSE confined himself to the case $(2,1)$. The general connex $(m, n)$ has been studied, in an elaborate memoir, by SintsoF. $\uparrow$

* Presented to the Society at the Evanston meeting, September 2, 1902. Received for publication April 11, 1903.

†Ueber ein neues Grundgebilde der analytischen Geumetrie der Ebøne, Göttinger Nachrich. ten, 1872 ; reprinted, Mathematische Annalen, vol. 6 (1873), pp. 203-225.

$\ddagger$ C. Clebsch-Lindemanin, Vorlesungen über Geometrie, vol. 1 (1876), 2d section; Godt, Ueber den Connex erster Ordnung und zweiter Classe, Göttingen, 1873.

\$ Darboux, Bulletin des Sciences Mathematiques, ser. 2, vol. 2 (1878); STePhANOS, ibid., vol. 4 (1880).

\|Ueber ein Gebilde der analytischen Geometrie des Raumes, welche dem Connexe zwriter Ordnung und erster Classe entspricht, M a th e mati sch e A n nalen, vol. 14 (1879), pp. 294-322.

TTheory of the connex in space, in connection with the theory of partial differential equations of the first order (Russian), Publications of Kasan University, 1895, $254 \mathrm{pp.}$ This is known to the writer only through the author's summary in the Fortschritte der Mathematik. Cf. Theorie des connexes dans l'espace, Bulletin des Sciences Mathematiques, 1898, p. 176. 
A different extension to space is made in the present paper. We have namely, as the simple elements of space, not only the point and the plane, but also the line; for compound element we may take then not only the point-plane, but also the point-line or the plane-line.* The last combination however is merely the dual of the second, and therefore requires no separate study. The combination of a point and a line, represented analytically by the coördinates $\left(x_{1}, x_{2}, x_{3}, x_{4} ; p_{12}, p_{13}, p_{14}, p_{34}, p_{42}, p_{23}\right)$, will be termed simply an element. The total number of elements in space is $\infty^{7}$, so that in the corresponding geometry space is seven dimensional. An equation

$$
f(x ; p) \equiv a_{x}^{m} A_{p}^{m}=0,
$$

represents then a manifold of $\infty^{6}$ elements, which will be termed a point-line connex of space, or, when there is no ambiguity, simply a connex.

If we regard the point $x$ as fixed, the equation above is satisfied by $\infty^{3}$ lines, namely those lines which combined with the given point constitute elements of the connex; these lines form a complex (of $n$th order) which will be referred to as the complex corresponding to the point $x$. On the other hand, if the line $p$ is regarded as fixed, there are $\infty^{2}$ points which may be combined with it so as to give elements of the connex; these points form a surface (of $m$ th order) which we term the surface corresponding to the line $p$.

After the study of manifolds defined by a single equation $f(x ; p)=0$, we might consider the manifolds of smaller dimension defined by two or more equations. Thus two equations $f_{m, n}=0, f_{m^{\prime}, n^{\prime}}^{\prime}=0$ represent $\infty^{5}$ elements, namely, those common to the corresponding connexes $(m, n),\left(m^{\prime}, n^{\prime}\right)$. To each point there corresponds, in this manifold, a congruence of lines, and to each line, a curve. The order of the congruence is $n n^{\prime}$ and the order of the curve is $m m^{\prime}$.

Three equations $f_{m, n}=0, f_{m^{\prime}, n^{\prime}}^{\prime}=0, f_{m^{\prime \prime}, n^{\prime \prime}}^{\prime \prime}=0$ represent a manifold of $\infty^{4}$ elements. The lines corresponding to a given point constitute a regulus of order $2 n n^{\prime} n^{\prime \prime}$; and the points corresponding to a given line form a discrete set, the number being $\mathrm{mm}^{\prime} \mathrm{m}^{\prime \prime}$. Four equations represent $\infty^{3}$ elements, etc.

Returning to the case of a single equation $f=0$ representing a connex, we proceed to define what may be termed, in analogy with CLEBSCH's terminology, its principal coincidence. This is composed of these elements of the connex for which the point $x$ and the line $p$ are mutually incident. Incidence of a point and line is a two-fold condition, whose complete expression requires, however, four equations $\dagger \Omega_{1}=\Omega_{2}=\Omega_{3}=\Omega_{4}=0$. The principal coincidence consists then of the $\infty^{4}$ elements satisfying the equations

$$
f=0, \quad \Omega_{1}=\Omega_{2}=\Omega_{3}=\Omega_{4}=0 .
$$

* To complete the series, another compound element must be considered, vamely the combination of a point, a line, and a plane. This the author expects to do elsewhere.

† See § 4, equations (13). 
To each point there corresponds a single infinity of lines passing through it, forming a conical surface. This suggests an intimate relation between the geometry of point-line connexes and the theory of total or MONGE * differential equations in three variables, which the author expects to discuss elsewhere.

The object of the present paper is to study the simplest and most important case of the new type of connex, namely the case $(1,1)$. This is defined by a bilinear quaternary-senary form equated to zero. The complex corresponding to a point is now linear, and the surface corresponding to a line reduces to a plane. We have thus two linear transformations, one from a point to a linear complex, and the other from a line to a plane. These are discussed in $\S \S 1-3$ from the point of view of distinct spaces, and in $\S \S 3-6$ from that of superposed spaces, the last section being devoted to the principal coincidence. In the next section a covariant point-plane connex of type $(2,1)$ is derived. Normal forms with respect to both digredient and cogredient transformations are deduced in $\S 9$ and $\S 11$.

The basis of a purely synthetic treatment is furnished in $\S 8$, where it is shown that the bilinear connex is equivalent to a correspondence between the points of a quadric surface and the lines of a linear congruence. This suggests the isomorphism of certain well-known projective groups, and the equivalence of the corresponding geometries $(\S 10)$. In $\S 11$ it is shown that the connex is completely determined by its fundamental configuration of five arbitrary points and two arbitrary lines. The absolute invariants of the connex are obtained in $\S 12$.

\section{§1. The related linear transformations $F^{\prime}, F^{\prime \prime}$.}

The connex to be studied is defined by an equation of the type $\dagger$

$$
F \equiv \Sigma a_{i, k l} x_{i} P_{k l}=0,
$$

involving linearly the four point coördinates $x_{i}$ and the six line coördinates $P_{k l}$. Symbolically,

where

$$
F \equiv a_{x} A_{P},
$$

$$
\begin{aligned}
& a_{x} \equiv a_{1} x_{1}+a_{2} x_{2}+a_{3} x_{3}+a_{1} x_{4}, \\
& A_{P} \equiv A_{12} P_{12}+A_{13} P_{13}+A_{14} P_{14}+A_{34} P_{34}+A_{42} P_{42}+A_{23} P_{23},
\end{aligned}
$$

so that

$$
a_{i} A_{k l}=a_{i, k l}=-a_{i} A_{l k}=-a_{i, l k} .
$$

* Cf. Lie-Scheffens, Geometrie der Berïhrungstransformationen, Leipzig, 1896, chap. 7.

$\dagger$ 'Throughout the paper the indices $i, k, l$ are understood to take the values $1,2,3,4$, and the summations extend over all the indices involved. 
The $\infty^{2}$ points, each of which, combined with a fixed line $P$, gives an element of the connex, constitute a plane

$$
u_{i}=a_{i} A_{P}
$$

This will be termed the plane corresponding to the line $P$; and the linear transformation (2), which defines the passage from $P$ to $u$, will be called the transformation $F^{\prime}$.

Consider next, in connection with a fixed point $x$, the lines which may be combined with it so as to give elements of the connex. There are $\infty^{3}$ such lines constituting a linear complex $\Sigma L_{i k} P_{i k}=0$, the coefficients being

$$
L_{i k}=A_{i k} a_{x} \text {. }
$$

This is the linear complex corresponding to the point $x$; the equations (3) define the linear transformation from $x$ to $L$, which we shall refer to as the transformation $F^{\prime \prime}$.

We then have, in connection with the general bilinear connex $F$, two linear transformations $F^{\prime}$ and $F^{\prime \prime}$, each of which determines the other and also $F$. The study of $F$ may therefore be regarded as equivalent to that of either of the transformations (2) or (3).

\section{§2. The transformation $F^{\prime}$ and the two fundamental lines.}

The transformation (2), written out in extenso, is

$$
\begin{aligned}
& u_{1}=a_{1,12} P_{12}+a_{1,13} P_{13}+a_{1,14} P_{14}+a_{1,34} P_{34}+a_{1,42} P_{42}+a_{1,23} P_{23}, \\
& u_{2}=a_{2,12} P_{12}+a_{2,13} P_{13}+a_{2,14} P_{14}+a_{2,34} P_{34}+a_{2,42} P_{42}+a_{2,23} P_{23}, \\
& u_{3}=a_{3,12} P_{12}+a_{3,13} P_{13}+a_{3,14} P_{14}+a_{3,34} P_{34}+a_{3,42} P_{42}+a_{3,23} P_{23}, \\
& u_{4}=a_{4,12} P_{12}+a_{4,13} P_{13}+a_{4,14} P_{14}+a_{4,34} P_{34}+a_{4,42} P_{42}+a_{4,23} P_{23} .
\end{aligned}
$$

The plane $u$, into which any line $P$ is transformed, is unique unless all its coördinates vanish, i. e., unless

$$
a_{1} A_{P}=a_{2} A_{P}=a_{3} A_{P}=a_{4} A_{P}=0 .
$$

These linear equations define two lines, say $\Pi^{\prime}, \Pi^{\prime \prime}$, so that we may write

$$
a_{i} A_{\Pi^{\prime}}=0, \quad a_{i} A_{\Pi^{\prime \prime}}=0 .
$$

The transformation $F^{\prime}$ thus possesses two fundamental lines $\Pi^{\prime}, \Pi^{\prime \prime}$ having the property that the corresponding plane is indeterminate.

In connection with the original connex $F$ the property of these lines may be restated: Each of the fundamental lines combined with an arbitrary point forms an element belonging to $F$. 
The correspondence between the lines $P$ and the planes $u$, defined by (4), is not uniquely reversible, as is evident from the fact that there are $\infty^{4}$ lines, but only $\infty^{3}$ planes. To each plane there corresponds indeed an infinity of lines whose locus we proceed to consider. The lines corresponding to $u$ satisfy the equations

$$
\frac{a_{1} A_{P}}{u_{1}}=\frac{a_{2} A_{P}}{u_{2}}=\frac{a_{3} A_{P}}{u_{3}}=\frac{a_{4} A_{P}}{u_{4}} .
$$

These equations define a regulus * (half-quadric) which, in virtue of (6), contains the lines $\Pi^{\prime}$ and $\Pi^{\prime \prime}$.

Conversely, consider the regulus determined by a given line $P$ and the fundamental lines. The coördinates of any line of this regulus are of the form

$$
\lambda P_{i k}+\lambda^{\prime} \Pi_{i k}+\lambda^{\prime \prime} \Pi_{i k}^{\prime \prime}
$$

The corresponding plane has the coördinates

$$
a_{i} A_{\lambda P+\lambda^{\prime} \Pi^{\prime}+\lambda^{\prime \prime} \Pi^{\prime \prime}}=\lambda a_{i} A_{P}+\lambda^{\prime} a_{i} A_{\Pi^{\prime}}+\lambda^{\prime \prime} a_{i} A_{\Pi^{\prime \prime}},
$$

which by (6) reduce simply to $\lambda a_{i} A_{P}$, namely the coördinates of the plane corresponding to $P$. The result obtained may be stated as follows :

The lines of any regulus which contains the fundamental lines $\Pi^{\prime}, \Pi^{\prime \prime}$ all transform by $F^{\prime}$ into the same plane. Conversely, corresponding to any plane $u$, there are an infinite number of lines, constituting a regulus passing through $\Pi^{\prime}, \Pi^{\prime \prime}$.

§ 3. The transformation $F^{\prime \prime}$ and the quadric $\omega$.

Written out in extenso, the second transformation, defined by (3), becomes

$$
\begin{aligned}
& L_{12}=a_{1,12} x_{1}+a_{2,12} x_{2}+a_{3,12} x_{3}+a_{4,12} x_{4}, \\
& L_{13}=a_{1,13} x_{1}+a_{2,13} x_{2}+a_{3,13} x_{3}+a_{4,13} x_{4}, \\
& L_{14}=a_{1,14} x_{1}+a_{2,14} x_{2}+a_{3,14} x_{3}+a_{4,14} x_{4}, \\
& L_{34}=a_{1,34} x_{1}+a_{2,34} x_{2}+a_{3,34} x_{3}+a_{4,34} x_{4}, \\
& L_{42}=a_{1,42} x_{1}+a_{2,42} x_{2}+a_{3,42} x_{3}+a_{4,42} x_{4}, \\
& L_{23}=a_{1,23} x_{1}+a_{2,23} x_{2}+a_{3,23} x_{3}+a_{4,23} x_{4} .
\end{aligned}
$$

Here the quantities $L$ are the coördinates of the linear complex corresponding to the point $x$, so that the complex itself is

$$
L_{P} \equiv \Sigma L_{i k} P_{i k}=0 .
$$

* The term regulus hereafter will be used in a special sense, denoting the system defined by three linear equations, that is, one set of generators of a quadric surface. Cf. the French term demi-quadrique. 
The complex will be special only if its invariant

$$
[L, L] \equiv 2\left(L_{12} L_{34}+L_{13} L_{42}+L_{14} L_{23}\right)
$$

vanishes. Substituting the values of $L$, the condition takes the form

$$
\omega \equiv \omega_{x}^{2} \equiv\left[A, A^{\prime}\right] a_{x} a_{x}^{\prime}=0,
$$

where

$$
\left[A A^{\prime}\right]=A_{12} A_{34}^{\prime}+A_{13} A_{42}^{\prime}+A_{14} A_{23}^{\prime}+A_{34} A_{12}^{\prime}+A_{42} A_{13}^{\prime}+A_{23} A_{14}^{\prime} .
$$

Therefore,

The points which are transformed into special linear complexes form a quadric surface $\omega=0$.

The transformation $F^{\prime \prime}$ produces, from all the points of space, $\infty^{3}$ linear complexes, which are now to be characterized. The six linear functions $A_{i k} a_{x}$ of the four variables $x_{i}$ must be connected by two linear relations. These are in fact

$$
L_{\Pi^{\prime}}=0, \quad L_{\Pi^{\prime \prime}}=0 .
$$

For if we substitute the values of $L$ in terms of $x$, we have for example $L_{\Pi^{\prime}}=a_{x} A_{\Pi^{\prime}}$, which vanishes by $(6)$.

The totality of linear complexes $L$, corresponding to all the points of space, constitute the linear three parameter system apolar * to $\Pi^{\prime}, \Pi^{\prime \prime}$.

This system includes $\infty^{2}$ special complexes, whose directrices are of course the tractors of the fundamental lines $\Pi^{\prime}, \Pi^{\prime \prime}$. These correspond to the points of the quadric $\omega . \dagger$

\section{$\S 4$ The five fundamental points $O_{\kappa}$.}

We have seen that to each point $x$, of the quadric $\omega$, there corresponds by $F^{\prime}$ a special linear complex; which may be regarded as equivalent to simply a line (its directrix). The coördinates of this line are

$$
P_{12}=A_{34} a_{x}, P_{13}=A_{42} a_{x} \text {, etc. }
$$

We inquire now concerning the points for which the corresponding line is an incident line, i. e., passes through the original point.

The general conditions expressing the incidence of a point $x_{i}$ and a line $p_{i k}$ are

$$
\begin{aligned}
\Omega_{1} & \equiv x_{2} p_{34}+x_{3} p_{42}+x_{4} p_{23}=0, \\
-\Omega_{2} & \equiv x_{3} p_{41}+x_{4} p_{13}+x_{1} p_{34}=0, \\
\Omega_{3} & \equiv x_{4} p_{12}+x_{1} p_{24}+x_{2} p_{41}=0, \\
-\Omega_{4} & \equiv x_{1} p_{23}+x_{2} \dot{p}_{31}+x_{3} p_{12}=0 .
\end{aligned}
$$

* In this connection the apolar relation signifies that the lines $\Pi^{\prime}, \mathrm{II}^{\prime \prime}$ are common to all the linear complexes $L$.

$\dagger$ For the detailed study of this correspondence see $\S 8$ below. 
The bilinear forms $\Omega_{i}$ are connected by the relation

$$
x_{1} \Omega_{1}+x_{2} \Omega_{2}+x_{3} \Omega_{3}+x_{4} \Omega_{4} \equiv 0 .
$$

Applying these conditions to the case of a point $x_{i}$ (of the surface $\omega$ ) and its corresponding line $P_{i k}$ as given by (12), we obtain

$$
\begin{aligned}
& q_{1} \equiv\left(x_{2} A_{12}+x_{3} A_{13}+x_{4} A_{14}\right) a_{x}=0, \\
& q_{2} \equiv\left(x_{3} A_{23}+x_{1} A_{24}+x_{1} A_{21}\right) a_{x}=0, \\
& q_{3} \equiv\left(x_{4} A_{34}+x_{1} A_{31}+x_{2} A_{32}\right) a_{x}=0, \\
& q_{4} \equiv\left(x_{1} A_{41}+x_{2} A_{42}+x_{3} A_{43}\right) a_{x}=0 .
\end{aligned}
$$

The quadrics $q_{i}$ are connected by the relation

$$
x_{1} q_{1}+x_{2} q_{2}+x_{3} q_{3}+x_{4} q_{4} \equiv 0 \text {. }
$$

The question now resolves itself into the discussion of the points common to the five quadrics

$$
\omega=0, \quad q_{1}=0, \quad q_{2}=0, \quad q_{3}=0, \quad q_{4}=0 .
$$

In the first place, the points common to the last four necessarily lie on the first. For by introducing the values $L_{i k}=A_{i k} a_{x}, q_{1}$ for example may be written

$$
L_{12} x_{2}+L_{13} x_{3}+L_{14} x_{4}=0 \text {. }
$$

As a consequence of the four equations $q_{i}=0$, we then have

which reduces to

$$
\left|\begin{array}{llll}
0 & L_{12} & L_{13} & L_{14} \\
L_{21} & 0 & L_{23} & L_{24} \\
L_{31} & L_{32} & 0 & L_{34} \\
L_{41} & L_{42} & L_{43} & 0
\end{array}\right|=0 \text {, }
$$

$$
L_{12} L_{34}+L_{13} L_{42}+L_{14} L_{23}=0 \text {; }
$$

this, however, in virtue of the values of $L_{i k}$, is simply another form of (11), the equation of the quadric $\omega=0$.

It remains to consider the points common to the four quadrics $q_{i}=0$. The quadrics $q_{1}=0, q_{2}=0, q_{3}=0$ intersect in 8 points. Any point common to these three, by $\left(14^{\prime}\right)$, will also lie on $q_{4}=0$, unless $x_{4}=0$. We must exclude then those among the 8 points which lie in the plane $x_{4}=0$. This plane cuts the first three quadrics in the conics

$$
\begin{aligned}
& \bar{q}_{1} \equiv\left(x_{2} A_{12}+x_{3} A_{13}\right) \bar{a}_{x}=0 \\
& \bar{q}_{2} \equiv\left(x_{3} A_{23}+x_{1} A_{21}\right) \bar{a}_{x}=0 \quad\left(\bar{a}_{x}=a_{1} x_{1}+a_{2} x_{2}+a_{3} x_{3}\right) \\
& \bar{q}_{3} \equiv\left(x_{1} A_{31}+x_{2} A_{32}\right) \bar{a}_{x}=0
\end{aligned}
$$


where the ternary forms are connected by

$$
x_{1} \bar{q}+x_{2} \bar{q}_{2}+x_{3} q_{3}=0 \text {. }
$$

It follows that these conics have 3 points in common; for of the 4 points in which the first two intersect, that point must be excluded for which $x_{3}=0$. We have then finally $8-3=5$ points common to the four quadrics $q_{i}=0$.

There are five points $O_{1}, O_{2}, O_{3}, O_{4}, O_{5}$ each of which $\left(O_{\kappa}\right)$ has the property that the linear complex which corresponds by $F^{\prime \prime}$ is special and has for directrix an incident line $\left(N_{\kappa}\right)$. These points lie on the quadric $\omega=0$.

\section{§5. The congruence $\Gamma$ and the surface $\sigma$.}

We return now to the transformation $F^{\prime}$ and inquire concerning the lines $P$ for which the corresponding plane $u$ is incident. The general conditions of incidence are

$$
\begin{aligned}
& u_{2} P_{12}+u_{3} P_{13}+u_{4} P_{14}=0, \\
& u_{3} P_{23}+u_{4} P_{24}+u_{1} P_{21}=0, \\
& u_{4} P_{34}+u_{1} P_{31}+u_{2} P_{32}=0, \\
& u_{1} P_{41}+u_{2} P_{42}+u_{3} P_{43}=0 .
\end{aligned}
$$

Substituting then $u_{i}=a_{i} A_{P}$, we obtain, as conditions on the lines $P$ in question,

$$
\begin{aligned}
& \Gamma_{1} \equiv\left(a_{2} P_{12}+a_{3} P_{13}+a_{4} P_{14}\right) A_{P}=0, \\
& \Gamma_{2} \equiv\left(a_{3} P_{23}+a_{4} P_{24}+a_{1} P_{21}\right) A_{P}=0, \\
& \Gamma_{3} \equiv\left(a_{4} P_{34}+a_{1} P_{31}+a_{2} P_{32}\right) A_{P}=0, \\
& \Gamma_{4} \equiv\left(a_{1} P_{41}+a_{2} P_{42}+a_{3} P_{43}\right) A_{P}=0 .
\end{aligned}
$$

The lines $P$, characterized by the property that the plane which corresponds by $F^{\prime}$ is incident, form a congruence $\Gamma$ defined by the equations (16).

What is the order and the class of this congruence? Consider any point, for convenience say $(1,0,0,0)$. If the line $P$ of the congruence passes through this point, then in the first place $P_{34}=P_{42}=P_{23}=0$; and in the second place the remaining three coördinates satisfy the equations

$$
\begin{aligned}
\left(a_{2} P_{12}+a_{3} P_{13}+a_{4} P_{14}\right)\left(A_{12} P_{12}+A_{13} P_{13}+A_{14} P_{14}\right) & =0, \\
& a_{1} P_{12}\left(A_{12} P_{12}+A_{13} P_{13}+A_{14} P_{14}\right)=0, \\
& a_{1} P_{13}\left(A_{12} P_{12}+A_{13} P_{13}+A_{14} P_{14}\right)=0, \\
& a_{1} P_{14}\left(A_{12} P_{12}+A_{13} P_{13}+A_{14} P_{14}\right)=0,
\end{aligned}
$$


obtained from (16). From the last three equations of this set, since $P_{12}, P_{13}, P_{14}$ cannot all vanish (for then all six coördinates would vanish), it follows that

$$
a_{1}\left(A_{12} P_{12}+A_{13} P_{13}+A_{14} P_{14}\right)=0,
$$

which, in combination with the first equation of (17), shows that there are two solutions. Since the coördinate system is entirely arbitrary, the point $(1,0,0,0)$ is really a point in general position, and it has thus been proved that through the general point there pass two lines of the congruence. The order of $\Gamma$ is therefore equal to two.

The class of the congruence is determined by finding the number of lines contained in a general plane, say $x_{1}=0$. We have now $P_{12}=P_{13}=P_{14}=0$; the first equation of (16) is then identically satisfied, and the other equations, yield three quadratic equations in $P_{32}, P_{42}, P_{23}$, whose discussion shows that there are three common solutions. The class of $\Gamma$ is therefore equal to three.

The congruence $\Gamma$ is of second order and third class. It follows that the number of congruence lines cutting a given line is equal to five.

To the double infinity of lines $\Gamma$ there corresponds, by $F^{\prime}$, a double infinity of planes which is now to be investigated. For this purpose, consider the equations (15) in connection with the system $\rho u_{i}=a_{i} A_{P}$, and eliminate the constant factor $\rho$ and the coördinates $P_{i k}$. The result may be written

$\left\|\begin{array}{ccccccc}a_{1,12} & a_{1,13} & a_{1,14} & a_{2,34} & a_{1,42} & a_{1,23} & u_{1} \\ a_{2,12} & \cdot & \cdot & \cdot & \cdot & \cdot & u_{2} \\ a_{3,12} & \cdot & \cdot & \cdot & \cdot & \cdot & u_{3} \\ a_{4,12} & \cdot & \cdot & \cdot & \cdot & \cdot & u_{4} \\ u_{2} & u_{3} & u_{4} & 0 & 0 & 0 & 0 \\ -u_{1} & 0 & 0 & 0 & -u_{4} & u_{3} & 0 \\ 0 & -u_{1} & 0 & u_{4} & 0 & -u_{2} & 0 \\ 0 & 0 & -u_{1} & -u_{3} & u_{2} & 0 & 0\end{array}\right\|=0$.

If we denote the determinants of seventh order, obtained by leaving out in turn the 5 th, 6 th, 7 th and 8 th row, by $\sigma_{1}, \sigma_{2}, \sigma_{3}$ and $\sigma_{4}$ respectively, it may be shown that these determinants are factorable. For example $\sigma_{4}$ vanishes if $u_{4}=0$, and therefore contains $u_{4}$ as a factor. The result of the detailed discussion is that there is a factor $\sigma$, of the third degree in $u$, which is common to all four determinants $\sigma_{i}$; so that

$$
\sigma \equiv \frac{\sigma_{1}}{u_{1}} \equiv \frac{\sigma_{2}}{u_{2}} \equiv \frac{\sigma_{3}}{u_{3}} \equiv \frac{\sigma_{4}}{u_{4}} .
$$

The planes which correspond to the lines of the congruence $\Gamma$ envelope a surface of third class $\sigma=O$, where $\sigma$ is defined by (19). 
$\S 6$. The principal coincidence and the covariant $K$.

The principal coincidence of the connex $F$ consists, by definition, of the $\infty^{4}$ incident elements belonging to $F$, i. e., of those elements in which the point and the lines are incident. Connected with each point $x$ there is a single infinity of lines $P$; these are the lines of the corresponding linear.complex $L$, defined in $\S 3$, which pass through $x$. These lines lie in a plane $U$, the polar plane of $x$ in the null system determined by $L$. The coördinates of $U$ are given by

$$
\begin{aligned}
& U_{1}=L_{12} x_{2}+L_{13} x_{3}+L_{14} x_{4}=\left(A_{12} x_{2}+A_{13} x_{3}+A_{14} x_{4}\right) a_{x}, \\
& U_{2}=L_{23} x_{3}+L_{24} x_{4}+L_{21} x_{1}=\left(A_{23} x_{3}+A_{24} x_{4}+A_{21} x_{1}\right) a_{x}, \\
& U_{3}=L_{34} x_{4}+L_{31} x_{1}+L_{32} x_{2}=\left(A_{34} x_{4}+A_{31} x_{1}+A_{32} x_{2}\right) a_{x}, \\
& U_{4}=L_{41} x_{1}+L_{42} x_{2}+L_{43} x_{3}=\left(A_{41} x_{1}+A_{42} x_{2}+A_{43} x_{3}\right) a_{x} .
\end{aligned}
$$

Comparing with (14), these may be abreviated

$$
U_{i}=q_{i}=q_{i x}^{2} .
$$

The principal coincidence is completely defined by this quadratic transformation from the point $x$ to the plane $U$. The transformation may also be expressed by means of the vanishing of a form involving two sets of point coördinates, namely by

$$
\begin{aligned}
K & \equiv k_{x}^{2} K_{X} \equiv \Sigma q_{i x}^{2} X_{i} \\
& \equiv\left\{\begin{array}{l}
\left(A_{12} x_{2}+A_{13} x_{3}+A_{14} x_{4}\right) X_{1}+\left(A_{23} x_{3}+A_{24} x_{4}+A_{21} x_{1}\right) X_{2} \\
+\left(A_{34} x_{4}+A_{31} x_{1}+A_{32} x_{2}\right) X_{3}+\left(A_{41} x_{1}+A_{42} x_{2}+A_{43} x_{3}\right) X_{3}
\end{array}\right\} a_{x}=0 .
\end{aligned}
$$

The vanishing of the covariant $K$ may therefore be interpreted: to each point $x$ there corresponds a plane $U$ (passing through it) containing the pencil of lines which, combined with $x$, constitute elements of the principal coincidence. The plane $U$ is the polar of $x$ with respect to the linear complex $L$ corresponding to $x$ by $F^{\prime \prime}$.

The plane $U$ ceases to be determined when the complex $L$ is special and has its directrix passing through $x$. This we have seen occurs only for the five points $O_{1}, O_{2}, O_{3}, O_{4}, O_{5}$ defined in $\S 3$. Therefore the principal coincidence of $F$ has five fundamental points $O_{1}, \ldots, O_{5}$; with each of these may be combined not merely a pencil of lines, but the entire bundle of lines through the point.

We consider now the inverse of the transformation defined by $(20)$ or $\left(20^{\prime}\right)$, and prove that there are in general three points $x$ which correspond to the same plane $U$. The points $x$ are in fact determined by the equations

$$
\frac{q_{1}}{U_{1}}=\frac{q_{2}}{U_{2}}=\frac{q_{3}}{U_{2}}=\frac{q_{4}}{U_{4}},
$$


or by the equivalent set

$$
U_{1} q_{2}-U_{2} q_{1}=0, \quad U_{1} q_{3}-U_{3} q_{1}=0, \quad U_{1} q_{4}-U_{4} q_{3}=0 .
$$

These quadratic equations in $x$, for all values of $U_{i}$, are satisfied by the coördinates of the five points $O_{k}$, since the latter cause all the quadrics $q_{i}$ to vanish. There are then only three variable solutions of the system-which proves the result stated above.

By means of $K=0$, to each point $X$ there corresponds a quadric surface

$$
K_{X} k_{x}^{2} \equiv X_{1} q_{1}+X_{2} q_{2}+X_{3} q_{3}+X_{4} q_{4}=0
$$

belonging to the linear system determined by $q_{1}, q_{2}, q_{3}, q_{4}$. This quadric is the locus of the point $x$ for which the corresponding plane $U\left(b y F^{\prime}\right)$ passes through the original point $X$.

The locus of the point $x$ whose corresponding plane $U$ is incident is the cubic surface $k_{x}^{2} K_{x}=0$. The locus of the point $X$ whose corresponding quadric surface is degenerate is the quartic surface

$$
\left(k, k^{\prime} k^{\prime \prime} k^{\prime \prime \prime}\right)^{2} K_{X} K_{X}^{\prime} K_{X}^{\prime \prime} K_{X}^{\prime \prime \prime}=0 \text {. }
$$

\section{§. The covariant point-plane connex $C$.}

Consider the bundle of lines through any point $X$; for each of these lines we have from (13)

$$
X_{2} P_{34}+X_{3} P_{42}+X_{4} P_{23}=0 \text {, etc. }
$$

To each line of the bundle there corresponds, by $F^{\prime}$, a plane $u$, so that

$$
a_{i} A_{P}-\rho u_{i}=0 \text {. }
$$

We prove now that the double infinity of planes so obtained themselves constitute a bundle.

Eliminate $\rho$ and the coördinates $P_{i k}$ from the four equations (23) and any three of the set (22). We obtain thus four relations, expressed by the vanishing of the determinants in the matrix

$\left|\begin{array}{ccccccc}a_{1,12} & a_{1,13} & a_{1,14} & a_{1,34} & a_{1,42} & a_{1,23} & u_{1} \\ a_{2,12} & \cdot & \cdot & \cdot & \cdot & \cdot & u_{2} \\ a_{3,12} & \cdot & \cdot & \cdot & \cdot & \cdot & u_{3} \\ a_{4,12} & \cdot & \cdot & \cdot & \cdot & \cdot & u_{4} \\ 0 & 0 & 0 & X_{2} & X_{3} & X_{4} & 0 \\ 0 & -X_{4} & X_{3} & -X_{1} & 0 & 0 & 0 \\ X_{4} & 0 & -X_{2} & 0 & -X_{1} & 0 & 0 \\ -X_{3} & X_{2} & 0 & 0 & 0 & -X_{1} & 0\end{array}\right|$


which result from omitting in turn the fifth, sixth, seventh and eighth row. These determinants have redundant factors $X_{1}, X_{2}, X_{3}, X_{4}$ respectively; the factor common to all is of the second degree in $X$ and of the first degree in $u$. The covariant thus obtained will be written

$$
C \equiv C_{X}^{2} u_{c} .
$$

We thus obtain a covariant point-plane connex $C=0$ of the second order and first class. In this connex, to each point $X$ there corresponds a bundle of planes, which coincides with the bundle obtained by $F^{\prime \prime}$ from the bundle of lines through $X$.

The coördinates of the vertex $x$ of this bundle are given by

$$
x_{i}=c_{i} C_{X}^{2} .
$$

If the point $x$ and the plane $u$ together form an element of the connex $C=0$, it follows from the above theorem that there exists a line through $X$ whose corresponding plane (by $F^{\prime}$ ) is $u$; or, what is equivalent, that of the $\infty$ lines forming a regulus corresponding by $F^{\prime}$ to $u$, one passes through $X$.

The quadric surface which corresponds, in the connex $C=0$, to a given plane $u$, contains the regulus of lines which are transformed by $F^{\prime \prime}$ into the same plane $u$.

The general point-plane connex of second order and first class has been investigated by Krause.* The results may be applied to the connex $C=0$, and thus indirectly to the original point-line connex $F$. For example, the locus of the plane $u$ for which the corresponding quadric is degenerate, is the surface of fourth class

$$
\left(C C^{\prime} C^{\prime \prime} C^{\prime \prime \prime}\right)^{2} u_{c} u_{c^{\prime}} u_{c^{\prime \prime}} u_{c^{\prime \prime \prime}}=0 ;
$$

the left member is therefore a covariant of $F$, involving the coefficients of $F$ to the sixteenth degree. The condition that the plane should be tangent to the corresponding quadric yields a surface of fifth class

$$
\left(C C^{\prime} C^{\prime \prime} u\right)^{2} u_{c} u_{c^{\prime}} u_{c^{\prime \prime}}=0 \text {. }
$$

There are 15 points $X$ each of which coincides with its corresponding point $x$.

The covariant $C$ was obtained by considering the $\infty^{2}$ lines passing through a point $X$. In an entirely analogous manner, by considering the $\infty^{2}$ lines lying in a plane $U$, we obtain another covariant, involving two sets of plane coördinates, which we write

$$
E \equiv U_{E}^{2} u_{e}
$$

By means of $E=0$, to a plane $U$ there corresponds a point $x$ such that the lines of $U$ are transformed, by $F^{\prime}$, into the planes through $x$; again to a plane $u$

* See reference on page 213 above. 
there corresponds a (class) quadric, containing the regulus of lines which $F^{\prime}$ transforms into $u$. It follows that the class quadric thus obtained is identical with the order quadric corresponding by $C=0$ to the same plane $u$.

\section{§8. A geometrical construction (or definition) of the connex. *}

If we interpret the $x_{i}$ as point coördinates in a space $s$, and the $P_{i k}$ as line coördinates in a distinct or superposed space $S$, then, connected with the general connex $F$, there is in $s$ a quadric surface $\omega$, and in $S$ a pair of lines $\Pi^{\prime}, \Pi^{\prime \prime}$, such that to each point of $\omega$ there corresponds a tractor of $\Pi^{\prime}, \Pi^{\prime \prime}$. The transformation from the point $x$ to the line $P$, as seen from (12), is linear, hence to a linear system of points corresponds a linear system of lines. But the only linear systems of points on $\omega$ are the generators; therefore to the points of a generator correspond lines forming a pencil. Since all the lines must be tractors of $\Pi^{\prime}, \Pi^{\prime \prime}$ it follows that the center of the pencil is a point on $\Pi^{\prime}$ or $\Pi^{\prime \prime}$ and that the plane of the pencil passes through $\Pi^{\prime \prime}$ or $\Pi^{\prime}$. Generators of the same system do not intersect; hence the corresponding pencils have centers on the same line $\Pi^{\prime}$ or $\Pi^{\prime \prime}$. Thus there are established $(1,1)$ correspondences between the generators of the first system on $\omega$ and the points of say $\Pi^{\prime}$; and between the generators of the second system and the points of $\Pi^{\prime \prime}$. The tractor corresponding to any point of $\omega$ is of course the line joining the points on $\Pi^{\prime}$ and $\Pi$ " which correspond respectively to the two generators passing through the given point.

If now we take arbitrarily, in the space $s$, a quadric $\omega$, and in the space $S$, a pair of lines $\Pi^{\prime}, \Pi^{\prime \prime}$, and establish $(1,1)$ correspondences between the two systems of generators and the points of $\Pi^{\prime}, \Pi^{\prime \prime}$ - or what is the same, establish a $(1,1)$ correspondence between the points of $\omega$ and the tractors of $\Pi^{\prime}, \Pi^{\prime \prime}$, we employ $9+4+4+6=23$ parameters. This is the number of parameters involved in the general connex $F$. It is thus rendered plausible that the connex is defined by the figure described.

The connex is in fact obtained most simply as follows, in terms of the related transformation $F^{\prime}$. Consider any line $P$ in the space $S$. The tractors conmon to $P, \Pi^{\prime}, \Pi^{\prime \prime}$ establish a homographic correspondence between the points of $\Pi^{\prime}$ and $\Pi^{\prime \prime}$; the corresponding generators must also be in $(1,1)$ correspondence; hence the locus of the points on $\omega$ corresponding to the tractors considered is a conic. Thus to the line $P$ in $S$ there corresponds finally a unique plane in $s$, namely the plane of the conic just obtained.

The transformation $F^{\prime \prime}$ is derived as follows. Consider any point $x$ in $s$, with the bundle of planes passing through it. Each of these planes intersects $\omega$ in a conic; this conic establishes a $(1,1)$ correspondence between the two systems of generators; the ranges on $\Pi^{\prime}$ and $\Pi^{\prime \prime}$ are thus placed in homographic

* This section furnishes the basis for a purely synthetic treatment of the bilinear connex. 
correspondence; hence the joins of corresponding points constitute a regulus (half-quadric). The $\infty^{2}$ planes of the bundle yield in this way $\infty^{2}$ reguli, and the $\infty^{3}$ lines of these reguli constitute a linear complex. This is the linear complex $L$ which corresponds to the point $x$ by means of the transformation $F^{\prime \prime}$

Given a plane $u$, it was seen in $\S 2$ that there is a regulus of lines which are all transformed by $F^{\prime \prime}$ into the same plane $u$. This regulus is now obtained as the conjugate regulus of that described in the preceding paragraph as derived from the conic in which $u$ cuts $\omega$.* $^{*}$

The connex $F$ itself is composed of $\infty^{6}$ elements $(x, P)$ each consisting of a point in $s$ and a line in $S$; these elements are characterized as follows: to the tractors of $P, \Pi^{\prime}, \Pi^{\prime \prime}$ correspond points on $\omega$ whose locus is a conic of which the plane passes through $x$.

The results of this section may be stated:

The study of the general bilinear connex is equivalent to the study of the configuration composed of a general quadric surface and a linear congruence (tractors of a pair of lines), the points of the surface and the lines of the congruence being placed in $(1,1)$ correspondence.

\section{§ 9. Normal form with respect to digredient transformation.}

From the preceding geometrical determination we may obtain a useful normal form. For this purpose it is sufficient to take the quadric $\omega$, the pair of lines $\Pi^{\prime}, \Pi^{\prime \prime}$, and the correspondence, in the simplest fashion. Let the quadric in $s$ be

$$
\omega \equiv x_{2} x_{3}-x_{1} x_{4}=0 ;
$$

its generators are

$$
\frac{x_{1}}{x_{2}}=\frac{x_{3}}{x_{4}}=\lambda, \quad \frac{x_{1}}{x_{3}}=\frac{x_{2}}{x_{4}}=\mu,
$$

where $\lambda, \mu$ are parameters. In $S$ let the two fundamental lines be

$$
\begin{array}{ll}
\Pi^{\prime}: & 0,0,1,0,0,0, \\
\Pi^{\prime \prime}: & 0,0,0,0,0,1 .
\end{array}
$$

The correspondence we define by assigning to the generator of the first system with parameter $\lambda$, the point $(\lambda, 0,0,1)$ on the line $\Pi^{\prime}$; and similarly, to the generator of the second system with parameter $\mu$, the point $(0, \mu, 1,0)$ on the

* From this it is seen that the regulus degenerates when, and only when, the plane $u$ is tangent to $\omega$; the result of the degeneration being two pencils having their vertices on $\Pi^{\prime}, \Pi^{\prime \prime}$ respectively. The totality of pencils so obtained constitute the quadratic complex

$$
\left(a \omega \omega^{\prime} \omega^{\prime \prime}\right)\left(a \omega^{\prime} \omega \omega^{\prime \prime}\right) A_{P} A_{P}^{\prime}=0,
$$

which therefore degenerates into the two linear complexes composed of the lines cutting $\Pi^{\prime}$ or $\Pi^{\prime \prime}$. 
line $\Pi^{\prime \prime}$. To the point $(\lambda, \mu)$ of $\omega$ there corresponds then the tractor joining the points $(\lambda, 0,0,1),(0, \mu, 1,0)$, namely the line

$$
\lambda \mu, \lambda, 0,-1, \mu, 0 .
$$

Consider any line $P_{i k}$ in $S$. If the tractor (30) is to intersect this line, then or, from $\left(28^{\prime}\right)$,

$$
P_{34} \lambda \mu+P_{42} \lambda+P_{13} \mu-P_{12}=0 ;
$$

$$
P_{34} x_{1}+P_{13} x_{2}+P_{42} x_{3}-P_{12} x_{4}=0 \text {. }
$$

This is in fact the plane corresponding to the line $P$, so that the transformation $F^{\prime}$ takes the form

$$
u_{1}=P_{34}, u_{2}=P_{13}, u_{3}=P_{42}, u_{4}=-P_{12} .
$$

The matrix of this transformation is

$$
\left|\begin{array}{rrrrrr}
0 & 0 & 0 & 1 & 0 & 0 \\
0 & 1 & 0 & 0 & 0 & 0 \\
0 & 0 & 0 & 0 & 1 & 0 \\
-1 & 0 & 0 & 0 & 0 & 0
\end{array}\right|,
$$

which, read by columns, gives for the transformation $F^{\prime \prime}$

$$
L_{12}=-x_{4}, L_{13}=x_{2}, L_{14}=0, L_{34}=x_{1}, L_{42}=x_{3}, L_{23}=0 .
$$

Finally the normal form of the connex is

$$
F \equiv x_{1} P_{34}+x_{2} P_{13}+x_{3} P_{42}-x_{4} P_{12}=0 \text {. }
$$

In the reduction to this form, it is assumed that $\omega$ is not degenerate and that $\Pi^{\prime}, \Pi^{\prime \prime}$ are distinct and non-intersecting. Consider first the condition that $\omega$ shall be degenerate, namely the vanishing of its discriminant $J$. We have

so that

$$
\omega \equiv \omega_{x}^{2} \equiv\left[A A^{\prime}\right] a_{x} a_{x}^{\prime},
$$

$$
\begin{aligned}
& J=\left(\omega \omega^{\prime} \omega^{\prime \prime} \omega^{\prime \prime \prime}\right)^{2}
\end{aligned}
$$

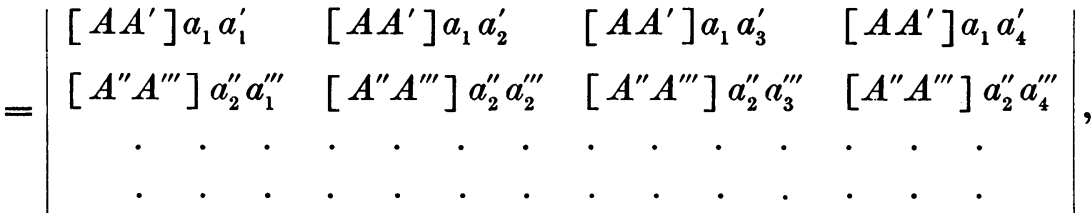

which, by permutation of the equivalent symbols, may finally be reduced to

$$
J=\left[A A^{\prime}\right]\left[A^{\prime \prime} A^{\prime \prime \prime}\right]\left[A^{(4)} A^{(5)}\right]\left[A^{(6)} A^{(7)}\right]
$$

$$
\left(a a^{\prime \prime} a^{(+)} a^{(6)}\right)\left(a^{\prime} a^{\prime \prime \prime} a^{(5)} a^{(7)}\right) .
$$


Consider in the second place the condition that $\Pi^{\prime}, \Pi^{\prime \prime}$ shall coincide. Since these are obtained as the lines common to four linear complexes, namely (20), the required condition is expressed by the vanishing of the combinant of these four complexes. This combinant is

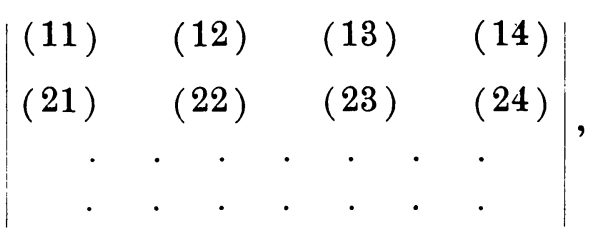

where (11), for example, denotes the invariant of $a_{1} A_{P}=0$, and (12), the simultaneous invariant of $a_{1} A_{P}=0, a_{2} A_{P}=0$; so that

$$
(11)=a_{1} a_{1}^{\prime}\left[A A^{\prime}\right], \quad(12)=a_{1} a_{2}^{\prime}\left[A A^{\prime}\right] \text {, etc. }
$$

Introducing these values, the combinant reduces to the invariant $J$ defined above. Therefore

When the quadric $\omega$ degenerates, the fundamental lines $\Pi^{\prime}, \Pi^{\prime \prime}$ coincide, and vice versa. The condition for either case is expressed by the vanishing of the invariant of the eighth degree $J$ defined by $\left(35^{\prime}\right) . *$

When $J$ does not vanish the initial representations (28), (28'), (29) are justified, and therefore

The connex $F$, by digredient transformation, may be reduced to the normal form (34), provided the invariant $J$ does not vanish. $\dagger$

\section{$\S 10$. The relation of certain projective groups.}

The discussion in $\S 8$ leads to the consideration of a $(1,1)$ correspondence between the points of quadric surface and the lines of a linear congruence. This correspondence is of interest in itself and suggests a connection between the related transformations which we shall now consider.

A proper quadric surface is transformed into itself by a well known six parameter group of collineations, composed of two continuous series $g_{6}, h_{6}$ distinguished by the fact that the transformations of the first kind turn each system of generators into itself, while those of the second kind interchange the two systems. On the other hand, a proper linear congruence, consisting of the tractors of two non-intersecting lines, is converted into itself by a seven parameter group of collineations $\left(G_{7}, H_{7}\right)$, the transformations of the first kind leaving each of the fundamental lines invariant while those of the second kind interchange them.

\footnotetext{
* To the points of $\omega$ correspond the congruence composed of the tractors of $\Pi^{\prime}, \Pi^{\prime \prime}$; the theorem above shows that the quadric and the congruence degenerate simultaneously.

$\dagger$ Moreover the reduction may be effected in $\infty^{7}$ distinct ways. This follows from the last theorem of $\S 10$.
} 
This group possesses a self-conjugate subgroup $I_{1}$ composed of the single infinity of skew perspectivities* which leave invariant the individual points of both fundamental lines.

In virtue of the correspondence between the elements, each transformation of the group $\left(G_{7}, H_{7}\right)$ induces a transformation of the points of $\omega$ which is equivalent to a collineation of the automorphic group $\left(g_{6}, h_{6}\right)$. The transformations of $I_{1}$ leave invariant each line of the congruence, and hence the induced transformation is merely identity. It follows that any two collineations of $\left(G_{7}, H_{7}\right)$ which may be obtained from each other by a transformation $I_{1}$ induce the same collineation of $\left(g_{6}, h_{6}\right)$.

The group $\left(g_{6}, h_{6}\right)$ of a proper quadric surface is isomorphic with the group $\left(G_{7}, H_{7}\right)$ of a proper linear congruence. The isomorphism is meriedric; to each transformation of the first group corresponds an infinity of transformations of the second, all of which are obtained from any one by means of the self-conjugate group $I_{1}$.

If we regard as identical, in the congruence group, those collineations which have the same effect upon the lines of the congruence (though they have different effects upon the points of space), the group reduces to one of six parameters $\left(\bar{G}_{6}, \bar{H}_{6}\right), \dagger$ and the relation above may be restated: The groups $\left(g_{6}, h_{6}\right)$ and $\left(\bar{G}_{6}, \bar{H}_{6}\right)$ are simply isomorphic.

The nature of the isomorphism is seen most clearly by observing that each point of the quadric, as well as each line of the congruence, may be represented by a pair of parameters $(\lambda, \mu)$, and that then both groups take the form

$$
\begin{aligned}
& \lambda^{\prime}=\frac{a \lambda+b}{c \lambda+d}, \quad \mu^{\prime}=\frac{\alpha \mu+\beta}{\gamma \mu+\delta} ; \\
& \lambda^{\prime}=\frac{a \mu+b}{c \mu+d}, \quad \mu^{\prime}=\frac{\alpha \lambda+\beta}{\gamma \lambda+\delta} .
\end{aligned}
$$

Another point of view is obtained by making use of the well known representation of lines by points in a space $R_{5}$. All the lines of space being represented by a manifold $M_{4}^{2}$, a linear congruence is represented by the intersection of this manifold with a 3 -flat, i. e., by an ordinary quadric surface.

The correspondence of elements and the isomorphism of groups justify the statement: Geometry on a quadric surface is equivalent to geometry in a linear congruence. Both may be adequately represented by means of double binary forms involving digredient variables. $\neq$

\footnotetext{
* Such a collineation is defined by the property that the join of corresponding points meets two fixed lines and is divided by them in a fixed anharmonic ratio.

T This is of course equivalent to the quotient group of $\left(G_{7}, H_{7}\right)$ over $I_{1}$.

$\ddagger \mathrm{Cf}$. E. KASNER, The cogredient and digredient theories of multiple binary forms, Transactions of the American Mathematical Society, vol. 4 (1903), pp. 86-102.

Trans. Am. Muth. Soc. 16
} 
The detailed study of the correspondence between the two geometries is of some interest and presents little difficulty. Thus to a conic corresponds an hyperboloid passing through the two fundamental lines; two conics intersect in two points, hence the corresponding hyperboloids intersect in two lines-in addition to the fundamental lines; to a twisted quartic of the first species corresponds a quartic surface having each of the fundamental lines for a double line, ${ }^{*}$ etc.

We return now to the connex $F$, and inquire concerning its automorphic transformations. The spaces $s$ and $S$ are here regarded as distinct, undergoing independent collineations, so that the number of constants involved in a transformation, made up of a pair of collineations, is thirty. The number of' conditions imposed by the invariance of $F$ is 23 , so that the automorphic group involves 7 parameters. The question is equivalent to the automorphic transformation of the configuration composed of the quadric $\omega$, the linear congruence defined by $\Pi^{\prime}, \Pi^{\prime \prime}$, and the correspondence between the two.

Consider any collineation $T$ of the group $\left(G_{7}, H_{7}\right)$. This in virtue of the correspondence induces in the space $s$ a collineation $t$ of the group $\left(g_{6}, h_{6}\right)$. The pair $t, T$ then constitute a transformation of the required type. Since one pair arises from each collineation of the group $\left(G_{7}, H_{7}\right)$ it follows that

The digredient transformations for which the general bilinear connex is invariant constitute a seven parameter group isomorphic with the group $\left(G_{7}, H_{7}\right)$ of the general linear congruence.

\section{§11. The fundamental configuration, and a normal form with respect to cogredient transformation.}

It has been seen in $\S 4$ that the bilinear connex has five fundamental points $O_{\kappa}$, for each of which the corresponding linear complex degenerates into an incident line $N_{\kappa}$. The configuration $O_{\kappa}, N_{\kappa}$ is not entirely arbitrary since the lines $N_{\kappa}$ are all tractors of the fundamental lines $\Pi^{\prime}, \Pi^{\prime \prime}$. There is however no other restriction, as is rendered plausible by an enumeration of constants; in fact the figure composed of five arbitrary points and two arbitrary lines involves $15+8$ constants, which is the number of constants involved in the general connex. We now prove precisely that

The connex is, in general, completely determined by the five arbitrary points $O_{1}, O_{2}, O_{3}, O_{4}, O_{5}$ and the two arbitrary lines $\Pi^{\prime}, \Pi^{\prime \prime}$.

We suppose that the points and lines above are in general position: specifi-

* The geometries above are also equivalent to the inversion geometry of the plane, so that to a circle corresponds an hyperboloid, etc. Cf. the author's article, The invariant theory of the inversion group: geometry on a quadric surface, Transactions of the A merican Mathematical Society, vol. 1 (1900), pp. 430-498. The results as to cyclic curves in chapter $\mathrm{V}$ of this article may easily be translated into theorems regarding ruled surfaces of the fourth order. 
cally, that the two lines do not intersect; that none of the points lies on either line; that no three of the points are collinear; and that none of the lines joining two of the points intersects both of the given lines. It follows from these assumptions that the lines $N_{\kappa}$, namely the tractors of $\Pi^{\prime}, \Pi^{\prime \prime}$ passing through the points $O_{\kappa}$, are all distinct and intersect $\Pi^{\prime}$ in distinct points $A_{\kappa}^{\prime}$, and $\Pi^{\prime \prime}$ in distinct points $A_{\kappa}^{\prime \prime}$. We proceed now with the proof of the theorem stated.

In the first place, the quadric surface $\omega$ is completely determined as follows: It must pass through the five points $O_{\kappa}$, and in such a way that the generators $g_{\kappa}^{\prime}$ of the first system passing through these points are homographic to the points $A_{\kappa}^{\prime}$, and that the generators $g_{\kappa}^{\prime \prime}$ of the second system are homographic to the points $A_{\kappa}^{\prime \prime}$. This is in fact equivalent to nine linear conditions. The quadric $\omega$ may be constructed synthetically quite simply. Take any non-degenerate quadric $\bar{\omega}$, and upon it any three points $\bar{O}_{1}, \bar{O}_{2}, \bar{O}_{3}$, no two lying on the same generator; denote the generators through these points by $\bar{g}_{1}^{\prime}, \bar{g}_{2}^{\prime}, \bar{g}_{3}^{\prime}$ and $\bar{g}_{1}^{\prime \prime}, \bar{g}_{2}^{\prime \prime}, \bar{g}_{3}^{\prime \prime}$. In the first* system find the two generators $\bar{g}_{4}^{\prime}, \bar{g}_{5}^{\prime}$ so that the five generators $\bar{g}_{\kappa}^{\prime}$ shall be homographic to the points $A_{\kappa}^{\prime}$; similarly find $\bar{g}_{4}^{\prime \prime}, \bar{g}_{5}^{\prime \prime}$. Denote the intersection of $\bar{g}_{4}^{\prime}, \bar{g}_{4}^{\prime \prime}$ by $\bar{O}_{4}$, and that of $\bar{g}_{5}^{\prime}, \bar{g}_{5}^{\prime \prime}$ by $\bar{O}_{5}$. Since the generators $\bar{g}_{4}^{\prime}, \bar{g}_{4}^{\prime \prime}$ are all distinct it follows that the points $\bar{O}_{\kappa}$ are all distinct and that no three are collinear. The same is true by hypothesis of the given points $O_{\kappa}$. There must exist then a unique collineation which transforms the points $\bar{O}_{\kappa}$ in the points $O_{\kappa}$. This collineation converts $\bar{\omega}$ into the required quadric $\omega$.

In the second place, there is a definite correspondence between the points of $\omega$ and the tractors of $\Pi^{\prime}, \Pi^{\prime \prime}$. For we have corresponding to $g_{1}^{\prime}, g_{2}^{\prime}, g_{3}^{\prime}$ the points $A_{1}^{\prime}, A_{2}^{\prime}, A_{3}^{\prime}$, and to $g_{1}^{\prime \prime}, g_{2}^{\prime \prime}, g_{3}^{\prime \prime}$, the points $A_{1}^{\prime \prime}, A_{2}^{\prime \prime}, A_{3}^{\prime \prime}$. This defines the correspondence; then in virtue of the above construction $A_{4}^{\prime}, A_{5}^{\prime}$ correspond to $g_{4}^{\prime}, g_{5}^{\prime}$, and $A_{4}^{\prime \prime}, A_{5}^{\prime \prime}$ correspond to $g_{4}^{\prime \prime}, g_{5}^{\prime \prime}$.

Having now determined the quadric $\omega$, the congruence defined by $\Pi^{\prime}, \Pi^{\prime \prime}$, and the correspondence between the two, it follows from $\S 9$ that the connex itself is uniquely determined, which completes the proof of the theorem.

It is of interest to carry out the argument on analytic lines as it leads to a normal form for $F$. Take for the coördinate tetrahedron that defined by the points $O_{1}, O_{2}, O_{3}, O_{4}$ and let $O_{5}$ be the unit point. The coördinates of the five fundamental points are thus
$O_{1}$
$\mathrm{O}_{2}$
$(1,0,0,0)$
$(0,1,0,0)$
$(0,0,1,0)$
$(0,0,0,1)$
$\mathrm{O}_{5}$
$(1,1,1,1)$.

Let the coördinates of $\Pi^{\prime}, \Pi^{\prime \prime}$ be $\lambda_{i k}^{\prime}, \lambda_{i k}^{\prime \prime}$ respectively. The problem is now to find the connex $F$ determined by these fundamental points and lines, i. e., to

\footnotetext{
* Which system is called first, is a matter of indifference ; for the interchange of the two systems, it may be shown, does not affect the final result.
} 
express the 24 coefficients $a_{i, k l}$ in terms of $\lambda_{i k}^{\prime}, \lambda_{i k}^{\prime \prime}$. The transformation $F^{\prime}$ carries the point $O_{1}$ into the line whose coördinates are

$$
a_{1,12}, \quad a_{1,13}, \quad a_{1,14}, \quad a_{1,34}, \quad a_{1,42}, \quad a_{1,23} ;
$$

since this is to be a line through $O_{1}$, it follows from the conditions for incidence that the last three coördinates vanish. Dealing similarly with $\mathrm{O}_{2}, \mathrm{O}_{3}, \mathrm{O}_{4}$, it is found that the matrix of $F$ is of the form

$$
\left|\begin{array}{llllll}
0 & 0 & 0 & a_{1,34} & a_{1,42} & a_{1,23} \\
0 & a_{2,13} & a_{2,14} & a_{2,34} & 0 & 0 \\
a_{3,12} & 0 & a_{3,14} & 0 & a_{3,42} & 0 \\
a_{4,12} & a_{4,13} & 0 & 0 & 0 & a_{4,23}
\end{array}\right| .
$$

The general connex contains 23 constants; by a proper collineation it should be possible to reduce the number to $23-15=8$. The matrix above, involving 12 homogeneous constants, is thus equivalent to only a semi-normal form of the connex. A reduced normal form is obtained by expressing the twelve coefficients of the matrix in terms of $\lambda_{i k}^{\prime}, \lambda_{i k}^{\prime \prime}$, which are in fact equivalent to only eight essential constants, since each sextuple of coördinates is connected by a quadratic relation. This reduction will now be carried out as follows.

The line into which $O_{1}$ is transformed, namely $\left(a_{1,34}, a_{1,42}, a_{1,23}, 0,0,0\right)$ is to be a tractor of $\Pi^{\prime}, \Pi^{\prime \prime}$, hence

$$
\begin{aligned}
& \lambda_{34}^{\prime} a_{1,34}+\lambda_{42}^{\prime} a_{1,42}+\lambda_{23}^{\prime} a_{1,23}=0, \\
& \lambda_{34}^{\prime \prime} a_{1,34}+\lambda_{42}^{\prime \prime} a_{1,42}+\lambda_{23}^{\prime \prime} a_{1,23}=0 .
\end{aligned}
$$

Similarly from $\mathrm{O}_{2}, \mathrm{O}_{3}, \mathrm{O}_{4}$ we find

$$
\begin{aligned}
& \lambda_{13}^{\prime} a_{2,13}+\lambda_{14}^{\prime} a_{2,14}+\lambda_{34}^{\prime} a_{2,34}=0 \\
& \lambda_{13}^{\prime \prime} a_{2,13}+\lambda_{14}^{\prime \prime} a_{2,14}+\lambda_{34}^{\prime \prime} a_{2,34}=0 ; \\
& \lambda_{12}^{\prime} a_{3,12}+\lambda_{14}^{\prime} a_{2,14}+\lambda_{42}^{\prime} a_{3,42}=0 \\
& \lambda_{12}^{\prime \prime} a_{3,12}+\lambda_{12}^{\prime \prime} a_{3,14}+\lambda_{42}^{\prime \prime} a_{3,42}=0 ; \\
& \lambda_{12}^{\prime} a_{4,12}+\lambda_{13}^{\prime} a_{4,13}+\lambda_{23}^{\prime} a_{4,23}=0 \\
& \lambda_{12}^{\prime \prime} a_{4,12}+\lambda_{13}^{\prime \prime} a_{4,13}+\lambda_{23}^{\prime \prime} a_{4,23}=0
\end{aligned}
$$

Finally, since $O_{5}$ is to be transformed into an incident line, we have the additional conditions

$$
\begin{aligned}
& a_{2,13}+a_{2,14}+a_{3,12}+a_{3,14}+a_{4,12}+a_{4,13}=0, \\
& a_{3,24}+a_{3,21}+a_{4,23}+a_{4,21}+a_{1,23}+a_{1,24}=0, \\
& a_{4,31}+a_{4,32}+a_{1,34}+a_{1,32}+a_{2,34}+a_{2,31}=0, \\
& a_{1,42}+a_{1,43}+a_{2,41}+a_{2,43}+a_{3,41}+a_{3,42}=0,
\end{aligned}
$$


of which, however, only three are independent. Taking then any three of this set with the eight equations above, we have eleven linear equations for the determination of the twelve homogeneous coefficients in terms of $\lambda_{i h}^{\prime}, \lambda_{i k}^{\prime \prime}$. The substitution of these values in the matrix (36) gives the reduced normal form of the matrix, and thus the final normal form of the connex $F$.

\section{$\S 12$ The absolute invariants of the connex.}

To obtain the absolute invariants of $F$, we may employ its covariant figures, in particular the quadric $\omega$, the five points $O_{k}$, the lines $\Pi^{\prime}, \Pi^{\prime \prime}$, and the tractors $N_{\kappa}$. The line $N_{\kappa}$ cuts $\Pi^{\prime}$ and $\Pi^{\prime \prime}$ in $A_{\kappa}^{\prime}$ and $A_{\kappa}^{\prime \prime}$; it intersects the quadric $\omega$ in the point $O_{\kappa}$ and in an additional point say $\bar{O}_{\kappa}$. Through $O_{\kappa}$ there pass two generators of $\omega$, say $g_{\kappa}^{\prime}, g_{\kappa}^{\prime \prime}$; and through $\bar{O}_{\kappa}$, two generators $\bar{g}_{\kappa}^{\prime}, \bar{g}_{\kappa}^{\prime \prime}$.

The number of independent absolute invariants of $F$ is $23-15=8$. These may be represented as anharmonic ratios by means of the covariant elements above. The five points $A_{\kappa}^{\prime}$ on $\Pi^{\prime}$ give two independent ratios, and so do the five points $A_{\kappa}^{\prime \prime}$ on $\Pi^{\prime \prime}$. In virtue of the $(1,1)$ correspondences between the points of $\Pi^{\prime}$ and the generators of the system $g^{\prime}$, and .between the points $\Pi^{\prime \prime}$ and the generators of the system $g^{\prime \prime}$, it follows that

$$
\begin{aligned}
& \left(g_{1}^{\prime}, g_{2}^{\prime}, g_{3}^{\prime}, g_{4}^{\prime}, g_{5}^{\prime}\right) \text { \& }\left(A_{1}^{\prime}, A_{2}^{\prime}, A_{3}^{\prime}, A_{4}^{\prime}, A_{5}^{\prime}\right), \\
& \left(g_{1}^{\prime \prime}, g_{2}^{\prime \prime}, g_{3}^{\prime \prime}, g_{4}^{\prime \prime}, g_{5}^{\prime \prime}\right) \text { \& }\left(A_{1}^{\prime \prime}, A_{2}^{\prime \prime}, A_{3}^{\prime \prime}, A_{4}^{\prime \prime}, A_{5}^{\prime \prime}\right),
\end{aligned}
$$

where the symbol denotes the equality of corresponding anharmonic ratios. Additional absolute invariants are obtained by considering however the generators $\bar{g}_{\kappa}^{\prime}, \bar{g}_{\kappa}^{\prime \prime}$, so that

$A$ set of eight independent absolute invariants is obtained by taking a pair of anharmonic ratios from each of the four quintuples $g_{\kappa}^{\prime}, g_{\kappa}^{\prime \prime}, \bar{g}_{\kappa}^{\prime}, \bar{g}_{\kappa}^{\prime \prime}$. All the absolute invariants of the connex are expressible in terms of these eight.

Another set is obtained by considering the points in which $\omega$ intersects $\Pi^{\prime}, \Pi^{\prime \prime}$. On each line $\Pi^{\prime}, \Pi^{\prime \prime}$ we have then seven points (two arising from $\omega$ and five from the tractors $N_{\kappa}$ ). Seven collinear points, however, have four independent anharmonic ratios, so that we obtain in all the requiresd eight.

Again, absolute invariants may be defined, though not so symmetrically, without making use of the quadric $\omega$. This must be possible, since it was seen in $\S 8$ that the fundamental figure $O_{\kappa}, \Pi^{\prime}, \Pi^{\prime \prime}$ by itself determines $F$. Construct first the tractors $N_{\kappa}$. Pass planes through each triple of fundamental points, cutting each of the remaining lines in two new points, and giving in all 20 points, four on each line $N_{\kappa}$. Thus with $O_{\kappa}, A_{\kappa}^{\prime}, A_{\kappa}^{\prime \prime}$ we have seven points on each of the five lines, and from these we derive 15 anharmonic ratios. These, of course, cannot be independent since they are expressible in terms of eight; but the relations between them are not of sufficient interest to be given here.

Columbia University, Nhw York. 